\title{
A GUITAR TABLATURE SCORE FOLLOWER
}

\author{
Robert Macrae, Simon Dixon \\ Centre for Digital Music, Queen Mary University of London \\ Mile End Road, E1 4NS, London \\ \{robert.macrae,simon.dixon\}@elec.qmul.ac.uk
}

\begin{abstract}
Although guitar tablature is the most prevalent musical score format on the internet, score following programs are only made for traditional musical scores or MIDI. The following work demonstrates a score following method for guitar tablature (tabs). In this method, a guitar tab parser interprets the score from HTML or ASCII text tabs which is then synthesised with guitar-like parameters. Finally, real-time audio to audio synchronisation methods are then used to accurately track the musicians position within the tab. A prototype application has been made to demonstrate this technique.
\end{abstract}

Keywords - Synchronisation, Score-following, Tablature

\section{INTRODUCTION}

Score following systems are traditionally associated with the automatic page turning of musical scores. However, on the internet, traditional musical scores are not the most common format used. Musical tabs indicate the instrumental fingering rather than musical pitches and ASCII text tab notation has become a popular way of sharing instructions for playing songs. The highest web ranked online guitar tab resource, www.ultimate-guitar.com, receives over 2 million unique viewers every month, ${ }^{1}$ significantly more than the 50 and 21 thousand the highest ranked musical score ${ }^{2}$ and $\mathrm{MIDI}^{3}$ websites receive, respectively. Furthermore, the desire for guitar based song learning using intuitive animated instructions is demonstrated in the popularity of the Guitar Hero series ${ }^{4}$ that has sold, to date, over 25 million copies of a game that involves pressing buttons to play animated notes on a guitar-like controller.

There are a number of score following systems that utilise different synchronisation techniques such as Dynamic Programming [1], Hidden Markov Models [2], and On-Line Time Warping [3]. However these systems are based on following either standard musical notation or MIDI files. It is easy to see why guitar tabs and chord charts have been mostly overlooked as a format for scores in the field of music information retrieval;

\footnotetext{
RM is funded by an EPSRC DTA Grant.

${ }^{1}$ http://siteanalytics.compete.com/ultimate-guitar.com+mididb.com+musicscores.com/

${ }^{2}$ www.music-scores.com

${ }^{3}$ www.mididb.com

${ }^{4}$ hub.guitarhero.com
}
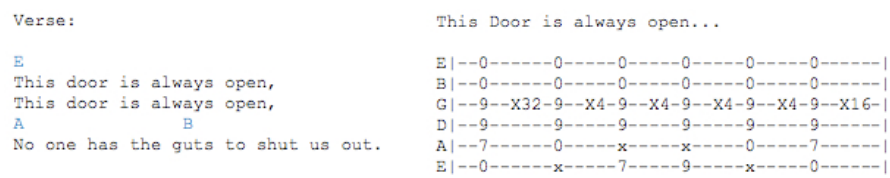

Fig. 1. Examples taken from two 'tablature' files showing the same song.

there is no standard format for writing tabs and often shortcuts are taken to simplify what is written with the expectation on the end-user to interpret the full score. However, for end-users this is a trivial process and reading tablature requires little/no musical training. Examples of two common formats are shown in Figure $1 .^{5}$ Authors are also known to invent their own additional notation and/or include comments to the reader throughout the score itself. A tab following system will need to correctly interpret a majority of the formats used for writing tabs, however, incomplete tabs are not considered for this tab following system as partial matching of scores represents a different problem area.

\section{METHODS}

The requirements for the proposed guitar tab score-following system consist of being able to interpret the musical score from the guitar tab and being able to calculate a musician's position within the piece in real-time. To interpret the tabs we implemented a text parsing system that extracts the musical score from the tab. Then, to automatically follow the guitar tab we implemented two systems, one that estimates the chord from the live audio of the musician and makes the alignment at the score level, and another that synthesises audio from the tab and makes the alignment with extracted audio features. An overview of the system is shown in Figure 2.

\subsection{Tab and HTML Parsing}

Tab parsing was implemented by using a set of rules to establish the nature of text lines within a tab. Firstly, non-tablature text

\footnotetext{
${ }^{5}$ Although strictly an abbreviation of tablature, we also use the term tab to refer to chord charts with/without lyrics (Figure 1) as is common in guitar tab websites.
} 




Fig. 2. Overview of the guitar tab following system.

and HTML are discarded so that only the tablature content remains. Lines analysed include chord definition lines (where the fret numbers for each string are displayed for a chord used in the song), chords and/or lyrics, tablature (where the string and fret numbers are shown for each note), capo changes, tuning definitions as well as intro, verse, chorus and bridge markers. The segmentation of these tab lines is then estimated so that combinations of lines, i.e. chords and lyrics on subsequent lines, are interpreted as being linked for simultaneous playback. From these tab lines, the musical events are extracted using the chord definitions and tuning where appropriate.

\subsection{Chord Estimation and Score Alignment}

A chord estimation was made using Stark and Plumbley's [4] technique for real-time chord recognition. This involves extracting a 12-bin chroma feature (based on the musical pitch class), followed by a classification technique that compares the chroma features to a number of binary templates to obtain chord labels. An established score pattern recognition system by Dannenberg [1] based on dynamic programming is then used to establish the most likely chord within the score that this interpreted chord represents.

\subsection{Tab Synthesis and Real-Time Synchronisation}

The tab is synthesised by converting the musical notes from the tab parser into MIDI notes and synthesising these with a guitar 'voice'. This gives an audible estimation of what the tab score would sound like. Real-time synchronisation involves extracting 12-bin chroma audio features from frames of the score audio and the recorded audio, and then using dynamic programming to find the link between these. The dynamic programming used to align these is based on Online Time Warping by Dixon [5] and can be seen in Figure 3.

\section{DEMONSTRATION}

An example application was created in Java with an interface designed in Max/MSP. ${ }^{6}$ A screenshot of the application can be seen in Figure 4. Within the application the user has the option of loading a MIDI file, copying tablature text directly into the
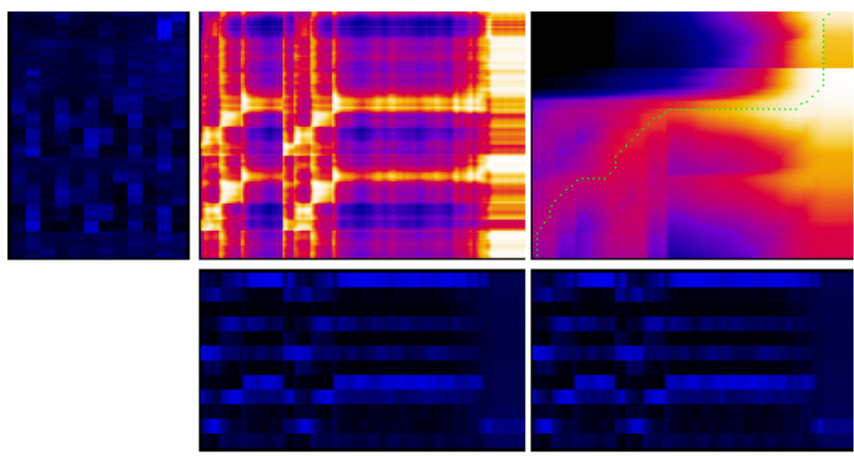

Fig. 3. Audio chroma vectors of a synthesised guitar and music showing (left) the similarity matrix and (right) the real-time DTW alignment

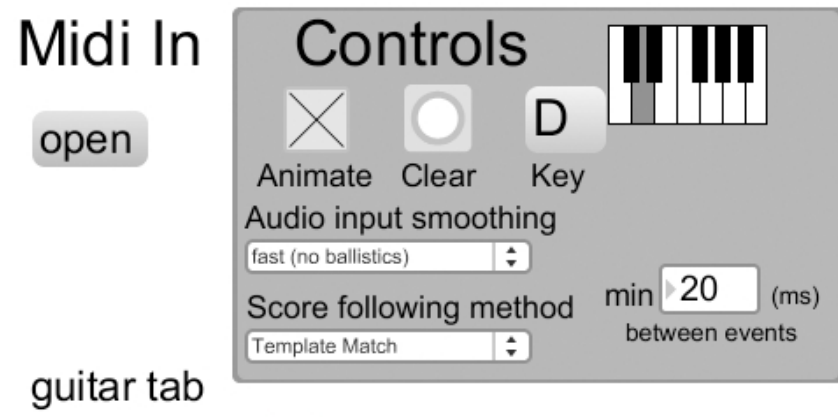

Fig. 4. Screenshot of the guitar tab score following system.

tab window or entering a URL in the window. The controls allow the user to have the score animated, change the musical key of the piece, select Template Matching or Real-time Dynamic Time Warping, and adjust the responsiveness of the synchronisation.

\section{REFERENCES}

[1] Roger Dannenberg, "An on-line algorithm for real-time accompaniment," in International Computer Music Conference, 1984.

[2] Arshia Cont and Diemo Schwarz, "Score following at Ircam," in MIREX Submission, 2006.

[3] Andreas Arzt, Gerhard Widmer, and Simon Dixon, "Automatic page turning for musicians via real-time machine listening," in Proceedings of the 18th European Conference on Artificial Intelligence, 2008.

[4] Adam Stark and Mark Plumbley, "Real-time chord recognition for live performance," in International Computer Music Conference, 2009.

[5] Simon Dixon, "Live tracking of musical performances using on-line time warping," in Proceedings of the 8th International Conference on Digital Audio Effects, 2005.

\footnotetext{
${ }^{6}$ http://cycling74.com/products/maxmspjitter/
} 